\title{
A variable frequency speed control system based on single-phase input without electrolytic capacitor
}

\author{
Danyang Bao, Yi Wang ${ }^{\mathrm{a}}$ and Ke Li \\ Department of Electrical Engineering, Harbin Institute of Technology Shenzhen Graduate School, Shenzhen, 518055
}

\begin{abstract}
A power control strategy based on PRN (Proportional Resonant and Inertia) is proposed in this paper. In the motor drive without dc-link electrolytic capacitor, the traditional PI controller is difficult to track the power which contains both ac and dc components. This characteristic cannot meet the system requirement of high input power factor. Therefore, PRN is used in power control. With higher open loop gain in both low frequency and specified resonant frequency, the PRN controller can provide an accurate tracking of the ac and dc power components. Then the input power factor and the phase error between the input current and the voltage can be improved obviously in the drive system. The simulation results verified the high power factor and low THD (total harmonic distortion) of the proposed method.
\end{abstract}

Keywords: dc-link electrolytic capacitor; power factor; PRN controller; power components.

\section{Introduction}

An electrolytic capacitors with high magnitude used for stabilizing the dc-link voltage leading to high cost, short life, large volume and hard to integrated of the conventional frequency control system. At the meanwhile, since the power factor is positively correlated to the diode conduction angle and the large electrolytic capacitor decreases the diode conduction angle, power utilization rate is reduced and the power factor correction effect is weakened [1,2]. Lots of efforts had been made to reduce the magnitude of electrolytic capacitors in the motor driven system.

H. Haga proposed a single-phase to three-phase power converter without the electrolytic capacitor. In [3], power control concept as a novel input current control method was proposed. It is explained using dither effect and adapted to the single-phase to three-phase circuit in this paper. But there are many current harmonics at the source side because of the strong power coupling between rectifier and inverter side. And in [4], current harmonics have been vastly improved by first-order repetitive control strategy. But THD is still too high to meet the guideline EN61000-3-2. Then in [5, 6], firstly, this paper proposes an inverter control method based on repetitive control in the q-axis current. Furthermore, this paper proposes a d-axis current control method, which reference is made in synchronous with the source voltage. The minimum THD of the proposed method obtained $17.6 \%$. The maximum power factor of the proposed method obtained 98.5\%. Otherwise, [7] proposed an ASD system using tapped inductor quasi-Z-source inverter without bulky electrolytic capacitor [6]. The proposed TL-QZSI can provide boost inversion ability with single stage power conversion, together with a voltage-buck freedom. But there are still many current harmonics at the source side and THD is

a Corresponding author : wangyi601@aliyun.com, missingdanyang@163.com 
$20 \%$.

This paper proposes a new power control method to reduce the THD and improve the input current harmonics in the variable frequency speed regulation system without electrolytic capacitor. Since with higher open loop gain in both low frequency and specified resonant frequency of the proposed controller, it also can overcome the problem of the traditional PI controller is hard to track the ac power component accurately. The performance of the proposed method is proved by the experimental results.

\section{Inverter power control strategy}

\subsection{Conventional inverter control method}

Large-capacity electrolytic capacitor increases the volume of the driver, reduces the life of the system and weakens the power correction effect. In order to increase the diode conduction angle and improve the power factor, the large-capacity electrolytic capacitor is replaced by a small capacity film capacitor. Film capacitor is mainly used to absorb the ripple current generated of switching frequency. The power converter is composed of the rectifier, film capacitors and inverter aspects of composition [3].

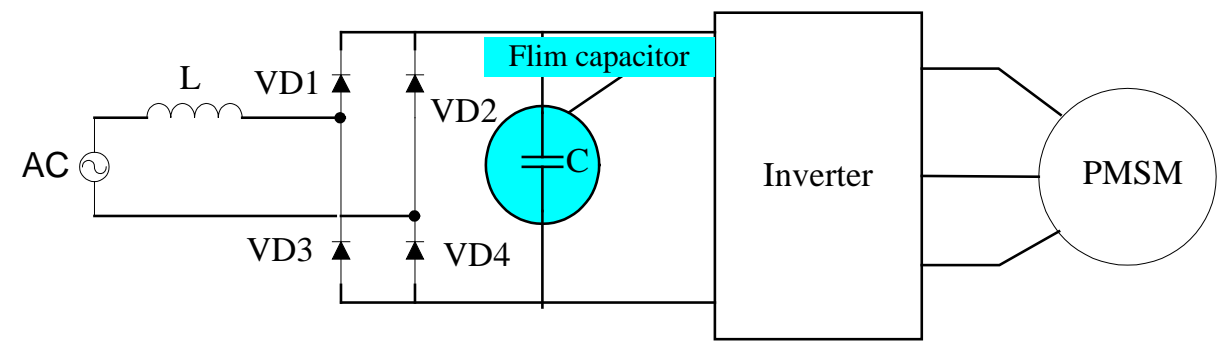

Figure 1. Electrolytic capacitors less inverter system

The system can't keep the stable dc-link voltage without a large capacity electrolytic capacitor. But the inertia of permanent magnet synchronous motors has the ability to absorb the pulsation of the single-phase power. Electrolytic capacitors-less inverter system makes DC voltage have some ripple to increase the diode conduction angle and improve the power factor [4].

Japanese scholars analysed the relationship between load transient power supply input voltage and current at unity power factor. By controlling the inverter output power controls to ripple with twice the synchronized the source voltage frequency to obtain high power factor $[4,5]$. When the input power factor is unit, the grid voltage, grid current, instantaneous load power is:

$$
\begin{gathered}
v_{\text {in }}=V_{\text {in }} \sin \omega_{\text {in }} t \\
i_{\text {in }}=I_{\text {in }} \sin \omega_{\text {in }} t \\
p=v_{\text {in }} \cdot i_{\text {in }}=P \sin ^{2} \omega_{\text {in }} t
\end{gathered}
$$

The input power can also be expressed as:

$$
p_{\text {in }}=k \cdot v_{\text {in }}^{2}
$$

Figure2 shows the block diagram for controlling the inverter output power. The input power command $p_{\text {in }}^{*}$ produced by the multiplication of speed controller output and $\sin ^{2} \omega_{i n} t p_{c}$ is dc-link capacitor power, $p_{i n v}^{*}$ is the inverter output power command. The difference between $p_{i n v}^{*}$ and $p_{i n v}$ is the inverter output power error. This error used in the PI controller, and its output is the q-axis current 
command $i_{q}^{*}$.

$$
\begin{gathered}
p_{c}=V_{d c} i_{c}=\frac{1}{2} \omega_{i n} C V_{d c}^{2} \sin \left(2 \omega_{i n} t\right) \\
p_{i n v}^{*}=p_{i n}^{*}-p_{c} \\
p_{i n v}=v_{d}^{*} \cdot i_{d}+v_{q}^{*} \cdot i_{q}
\end{gathered}
$$

\subsection{The problem of conventional inverter control method}

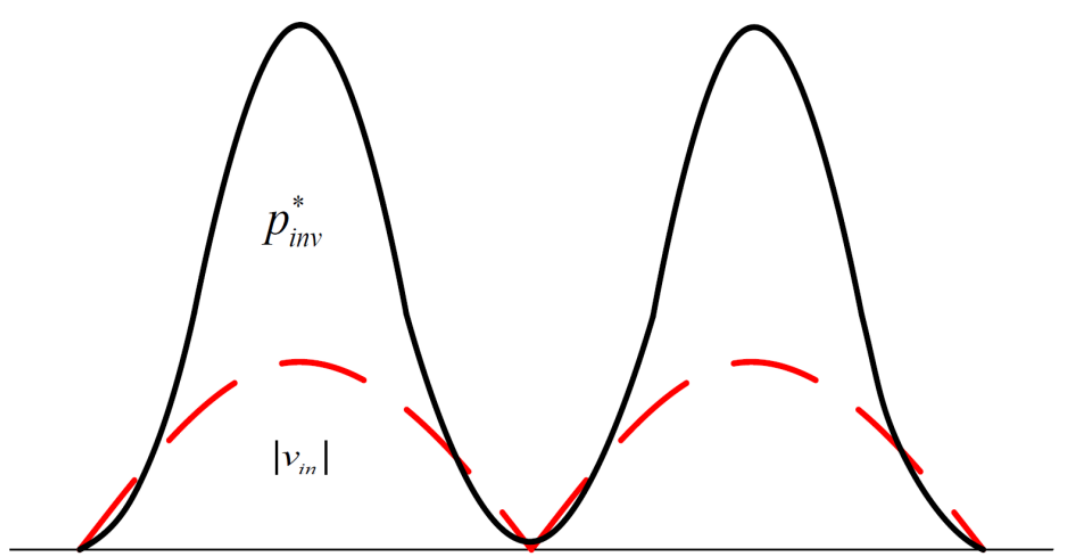

Figure 3. Schematic diagram of the relationship between Power and voltage waveform

The instantaneous power of load pulsates in 2 times of the grid voltage's frequency,and it includes both AC and DC components.

However, the traditional power control of the PI controller can only realize reliable tracking of the DC component. It is difficult to track the exchange amount and the grid voltage phase quickly and accurately.

Figure4 shows the bode diagram of PI controller with high gain at low frequencies. In order to realize the accurate tracking of power, it needs to address the problem of PI regulator in controlling the exchange amount.
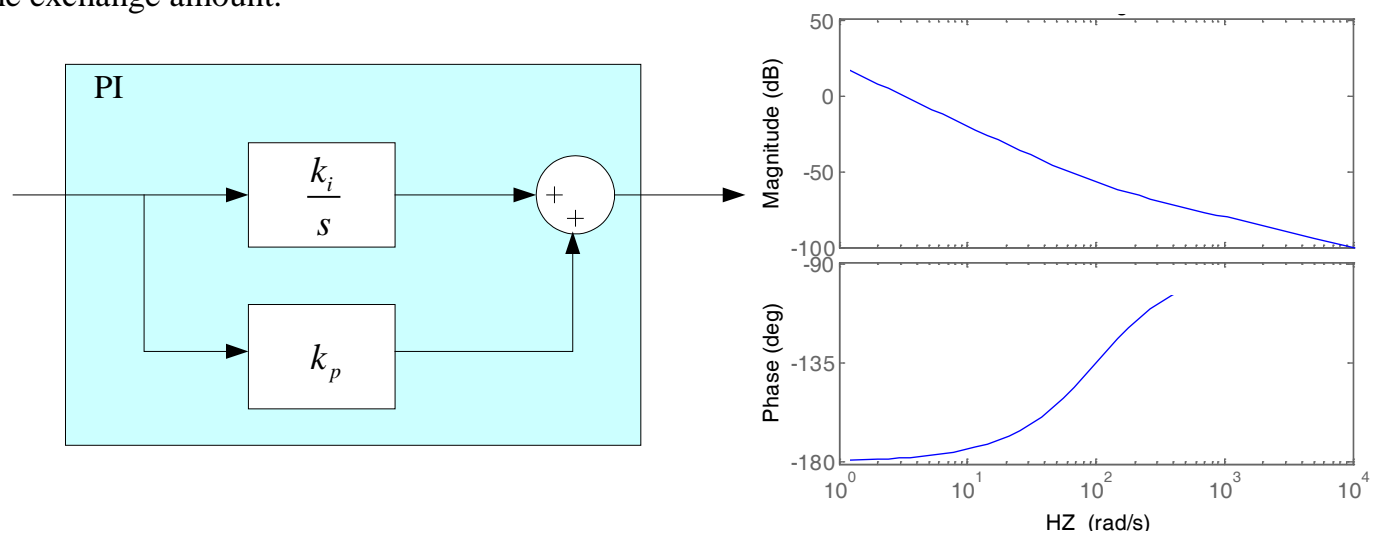

Figure 4. Bode diagram of the PI power controller 


\section{Proposed control method}

\subsection{The PR inverter power controller}

Compared with PI controller, PR (proportional resonant) controller is able to track a sinusoidal signal at a given frequency and the exchange amount [8]. $k_{p}$ is proportional gain, $k_{r}$ is resonance gain, $\omega_{0}$ is resonant frequency PR controller transfer function is:

$$
G_{P R}(\mathrm{~S})=k_{p}+\frac{2 k_{r} s}{s^{2}+\omega_{0}^{2}}
$$

Some scholars designed PR quasi-resonant controller to optimize PR controller and reduce the sensitivity of the frequency [9]. $\omega_{c}$ is cut-off frequency, its transfer function is:

$$
G_{P R}(S)=k_{p}+\frac{2 k_{r} \omega_{c} s}{s^{2}+2 \omega_{c} s+\omega_{0}^{2}}
$$

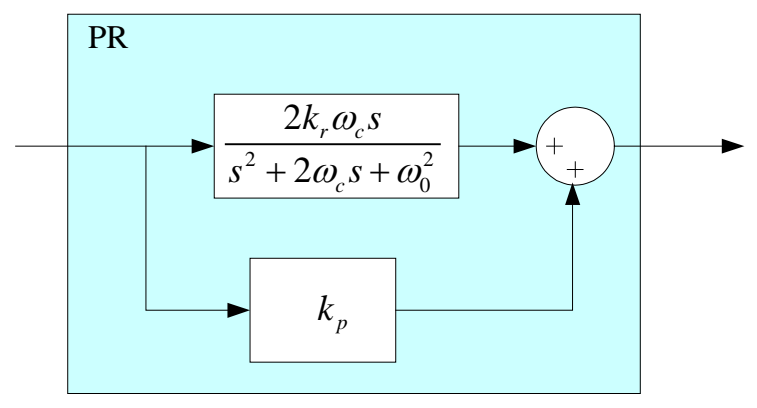

Figure5. Bode diagram of the PR power controller

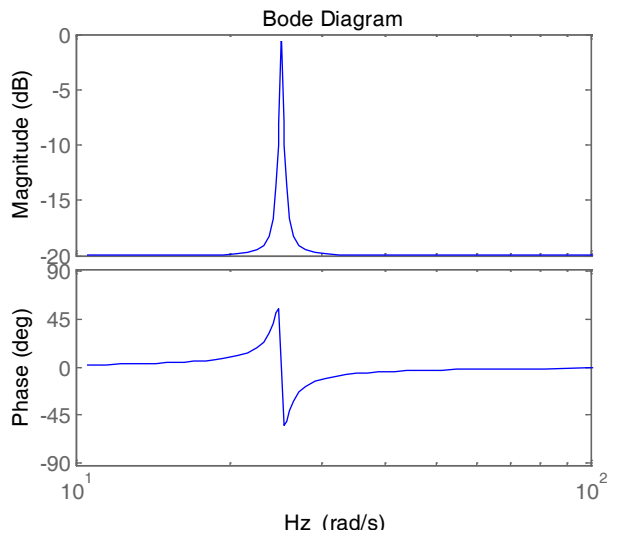

Figure5 shows the bode diagram of the PR controller. PR quasi resonant controller's frequency gain is still great at $\omega_{0}$, and the outer band $\omega_{0}$ high gain band is widened. But PR controller has small frequency gain in the low frequency. It attenuates the DC component of the inverter power and is not conducive to fast-track direct traffic.

It is necessary to control both DC component and AC component of power for the inverter system. Therefore, this paper investigates a new type of power controller control method which based on the above theories, achieves fast and accurate tracking of the system by effectively controlling the AC and the DC components.

\subsection{The proposed inverter power controller}

The new power controller consists of a proportional element, a resonant element and an inertia element (PRN). Under ideal conditions, the inertia link controls DC component, in the same time, the resonant elementcomes true tracking of AC component at $\omega_{0}$. The transfer functions as follows:

$$
G_{N}(s)=k_{p}+\frac{2 k_{r} \omega_{c} s}{s^{2}+2 \omega_{c} s+\omega_{0}^{2}}+\frac{k_{1}}{s+\lambda}
$$


Thus:

$$
\begin{gathered}
G_{N}(s)=\frac{k_{p} \cdot G_{1}(s)+2 k_{r} \omega_{c} \cdot G_{2}(s)+k_{1} \cdot G_{3}(s)}{G_{1}(s)} \\
G_{1}(s)=s^{3}+\left(2 \omega_{c}+\lambda\right) s^{2}+\left(2 \omega_{c} \cdot \lambda+\omega_{0}^{2}\right) s+\lambda \omega_{0}^{2} \\
G_{2}(s)=2 k_{r} \omega_{c}\left(s^{2}+\lambda s\right) \\
G_{3}(s)=k_{1} \cdot\left(s^{2}+2 \omega_{c} s+\omega_{0}^{2}\right)
\end{gathered}
$$

Where, $k_{1}$ is low pass gain constant, $\lambda$ is inertia coefficient. When $\omega_{0}=200 \pi \mathrm{rad} / \mathrm{s}, k_{r}=50$, $k_{1}=1, \omega_{c}=1 \mathrm{rad} / \mathrm{s}, \lambda=0.01$, the bode diagram of the new controller and PR controller transfer function is shown in Figure6:
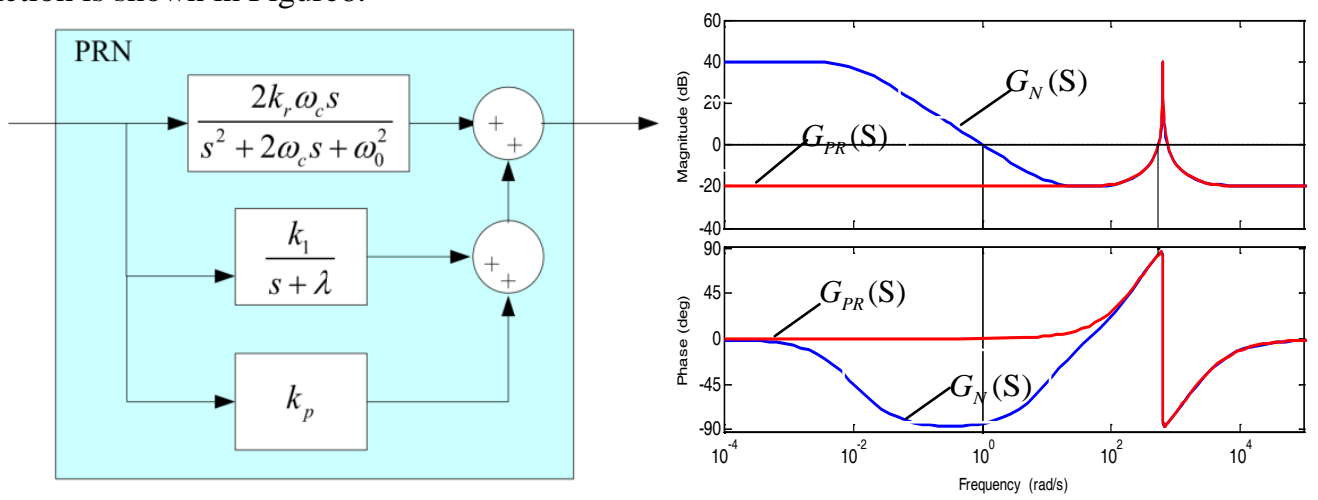

Figure 6. Bode diagram of the PRN and PR power controller

Figure6 shows the bode diagram of PRN and PR controller. New controller has a peak and approximate infinity at the resonant frequency $\omega_{0}$ and otherwise the signal gain will decrease. The phase angle of the resonant part from lead 90 degrees quickly reduced to lag 90 degrees to ensure accurate tracking. Besides, the new controller maintains a high-gain at low frequencies and reduces the decay rate at low-frequency components. Therefore, new controller ensures the AC and DC component accurate tracking at the same time.

\subsection{Effect on the new power controller of various parameters}

System parameters are significant to achieve fast-tracking power. By (10)-(14), the main parameters of the new power controller are $k_{1}, \lambda, \omega_{c}, k_{p}$ and $k_{r}$. Let $\omega_{0}=100 \pi \mathrm{rad} / \mathrm{s}, k_{r}=50, k_{1}=1$, $k_{p}=0.1$ which are constants. When $\lambda=0.01, \omega_{c}=0.1,1,10 \mathrm{rad} / \mathrm{s}$, the bode plots of $G_{N}(\mathrm{~s})$ is showed in Figure6, and $\omega_{\mathrm{c}}=1 \mathrm{rad} / \mathrm{s}, \lambda=0.01,0.1,1 \mathrm{rad} / \mathrm{s}$, the bode plots of $G_{N}(\mathrm{~s})$ is showed in Figure7: 


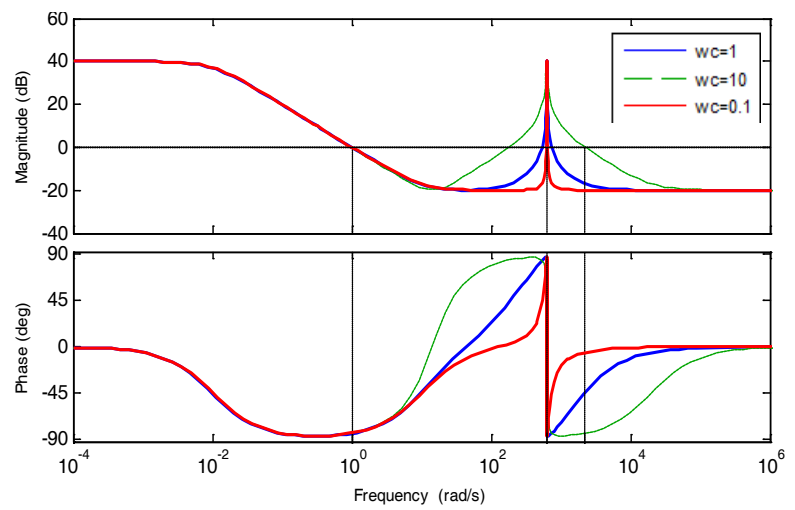

Figure 7. Bode diagram of the PRN at $\lambda=0.01, \omega_{c}=0.1,1,10 \mathrm{rad} / \mathrm{s}$

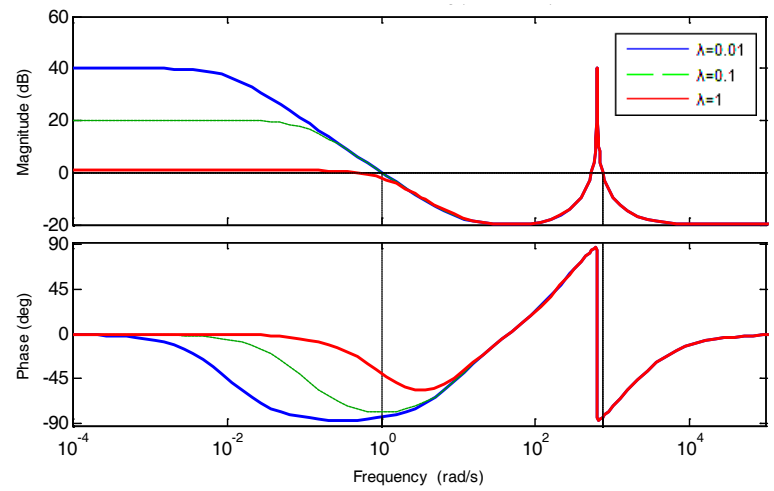

Figure 8. Bode diagram of the PRN at $\omega_{\mathrm{c}}=1 \mathrm{rad} / \mathrm{s}, \lambda=0.01,0.1,1 \mathrm{rad} / \mathrm{s}$

Figure7 and Figure8 show the bode diagram of PRN at different values of $\omega_{c}$ and $\lambda$.With $\omega_{c}$ increasing, phase angle rises slowly, and the time of lead-lag becomes longer. And gain amplitude decreases the frequency band is widened, the system sensitivity on the frequency will reduce. With $\lambda$ increasing, the low frequency gain reduces, the phase angle decreases attenuation and the input signal frequency sensitivity decreases.

\section{Simulation results}

This paper carries the simulation of the proposed method. The main motor parameters are as flows: $L_{S}=0.97 \mathrm{mH}, R_{s}=0.11 \Omega, p_{n}=4, J=0.0016 \mathrm{~kg} \cdot \mathrm{m}^{2}, \quad \psi_{f}=0.1119 \mathrm{~V} \cdot \mathrm{s}$. System parameters are $t=1 \mathrm{~s}, V=310 \mathrm{~V}, L=0.2 \mathrm{mH}, C=4.7 \mu \mathrm{F}$. The control block diagram of the electrolytic capacitors-less inverter system as figure9: 


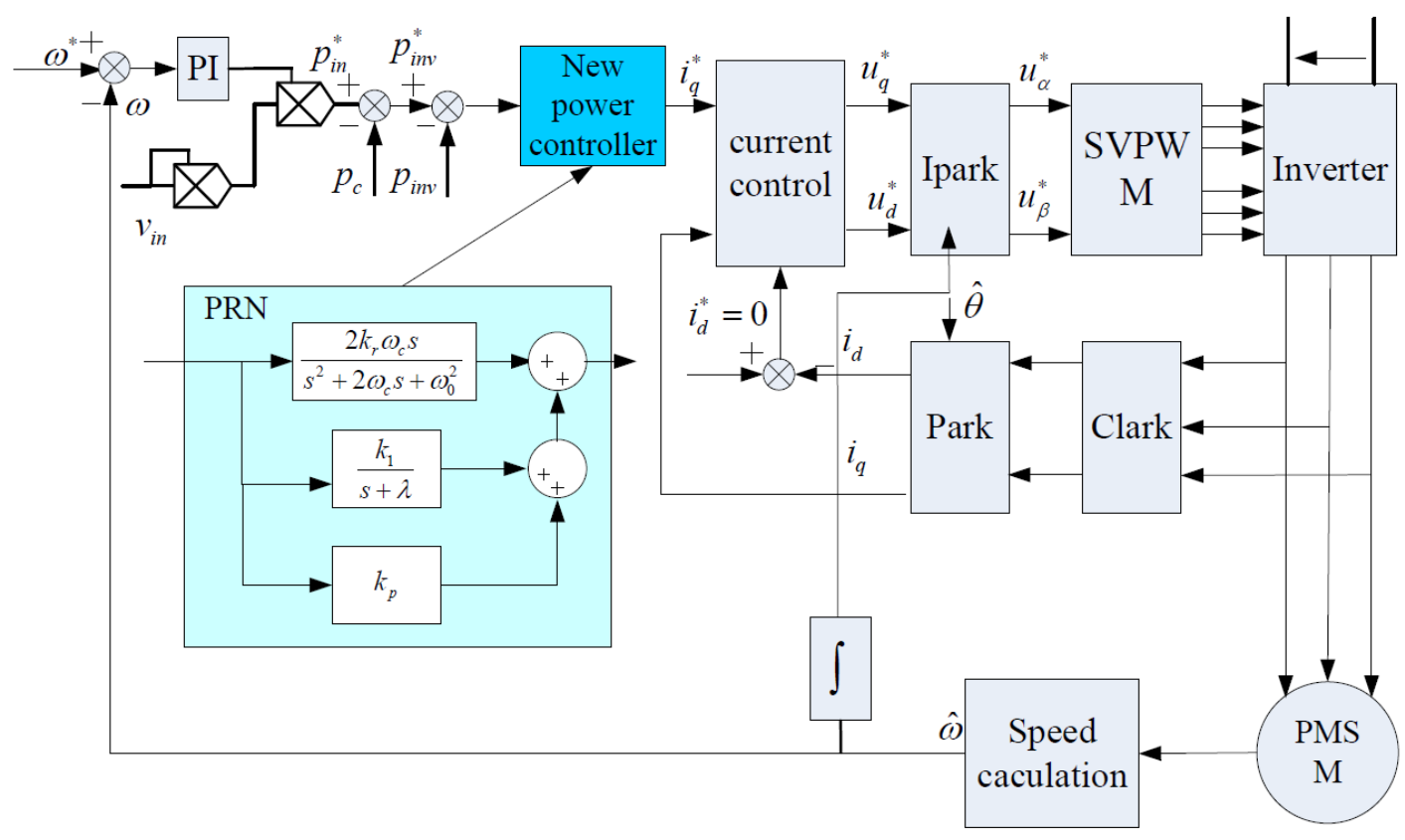

Figure 9. Control block diagram of the electrolytic capacitors less inverter system

Let $\omega_{0}=200 \pi \mathrm{rad} / \mathrm{s}, k_{r}=50, k_{1}=1, k_{p}=0.1, \lambda=0.1, \omega_{c}=1 \mathrm{rad} / \mathrm{s}$, based on the proportion rate of $\mathrm{AC}$ and $\mathrm{DC}$ component in inverter power. When $n=1000 \mathrm{r} / \mathrm{min}, T_{l}=5 \mathrm{~N} \cdot \mathrm{m}$. The simulation results of the new power controller are shown in flow figures:

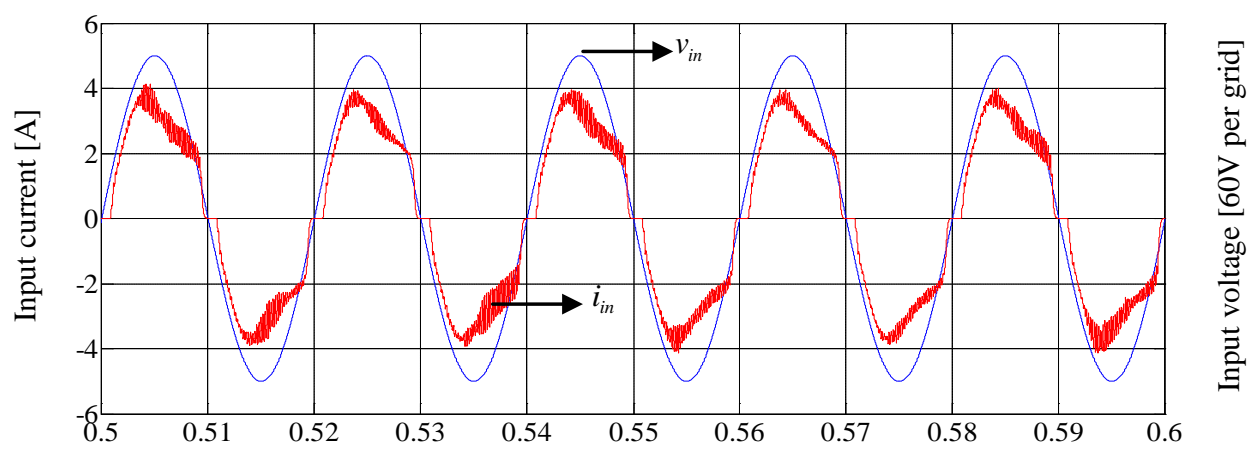

$t(\mathrm{~s})$

Figure 10. Simulation result of the traditional method of $v_{\text {in }}$ and $i_{\text {in }}$ 


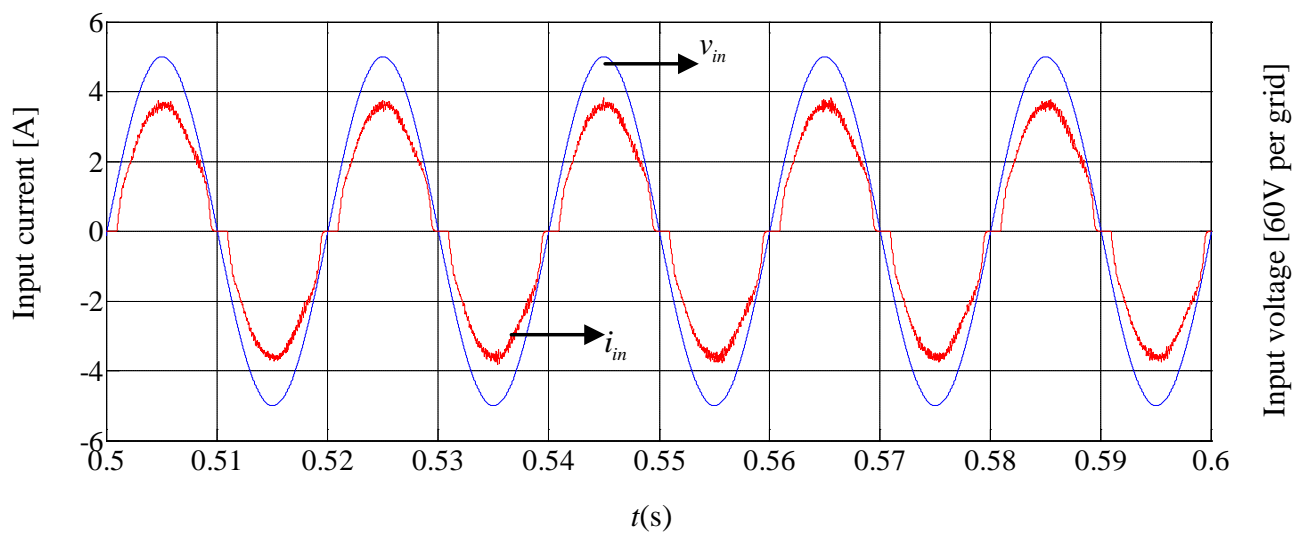

Figure 11. Simulation diagram of the proposed method of $v_{\text {in }}$ and $i_{\text {in }}$

Figure10 and figure11 show the simulation diagram of $v_{\text {in }}$ and $i_{\text {in }}$ by PRN and PI controller. In figure10, there are many current harmonics, waveform distorts seriously and the maximum power factor obtained by PI controller is $87.4 \%$. In figure 11, input current harmonic content reduces, waveform distortion decreases, and power factor improvesto $98.9 \%$.

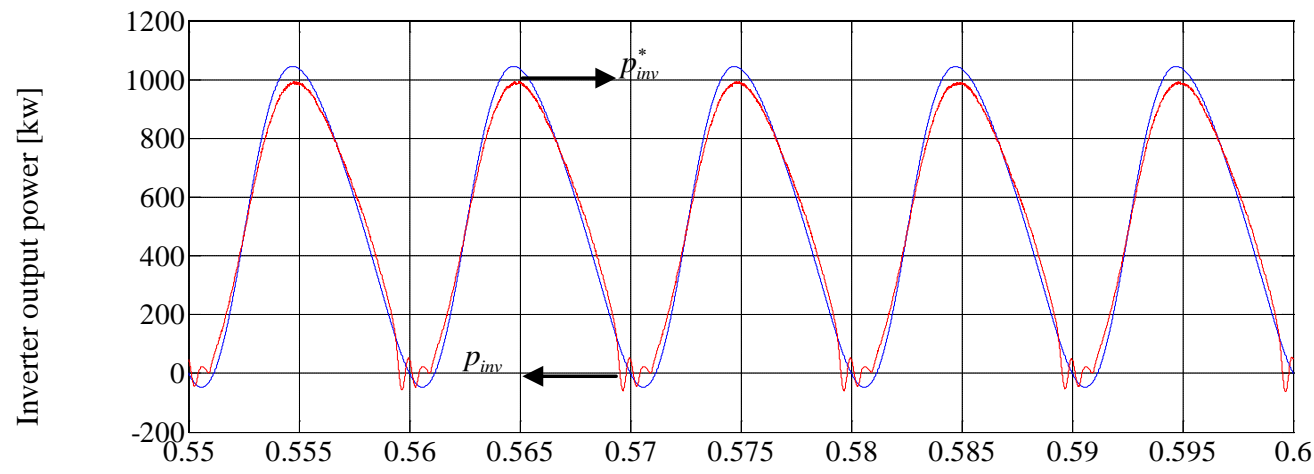

$t(\mathrm{~s})$

Figure 12. Simulation diagram of the traditional method of $p_{i n v}^{*}$ and $p_{i n v}$

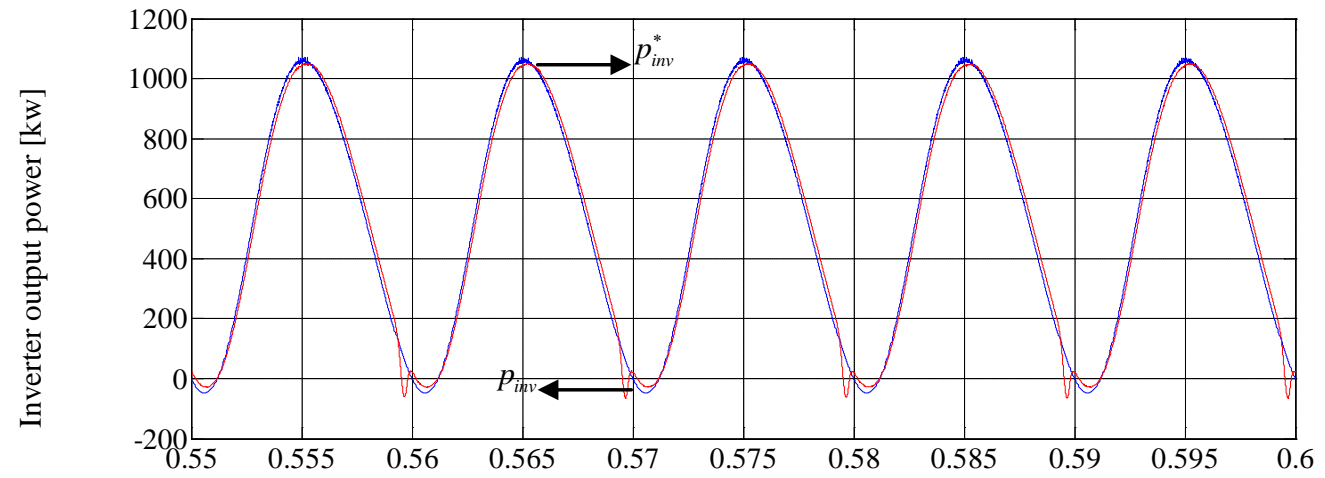

$t(\mathrm{~s})$

Figure 13. Simulation diagram of the proposed method of $p_{i n v}^{*}$ and $p_{i n v}$ 
Figure12 shows the simulation diagram of $p_{i n v}^{*}$ and $p_{i n v}$ in PI controller. Figure13 shows the simulation diagram of $p_{i n v}^{*}$ and $p_{i n v}$ in PRN controller. The effect of power tracking and system stability are further improved. Furthermore, this paper analyses THD of the input voltage and the input current to prove the effectiveness of the proposed method.

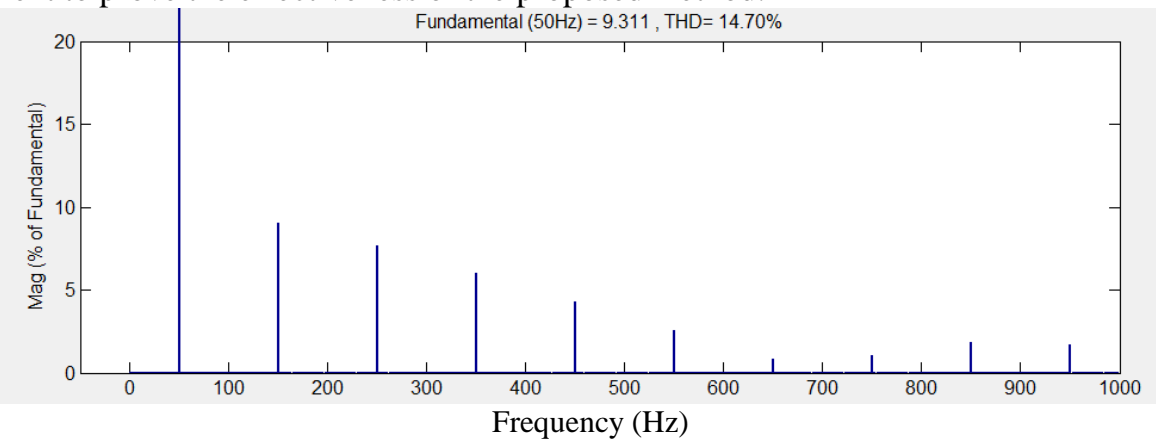

Figure 14. THD in the PR control method

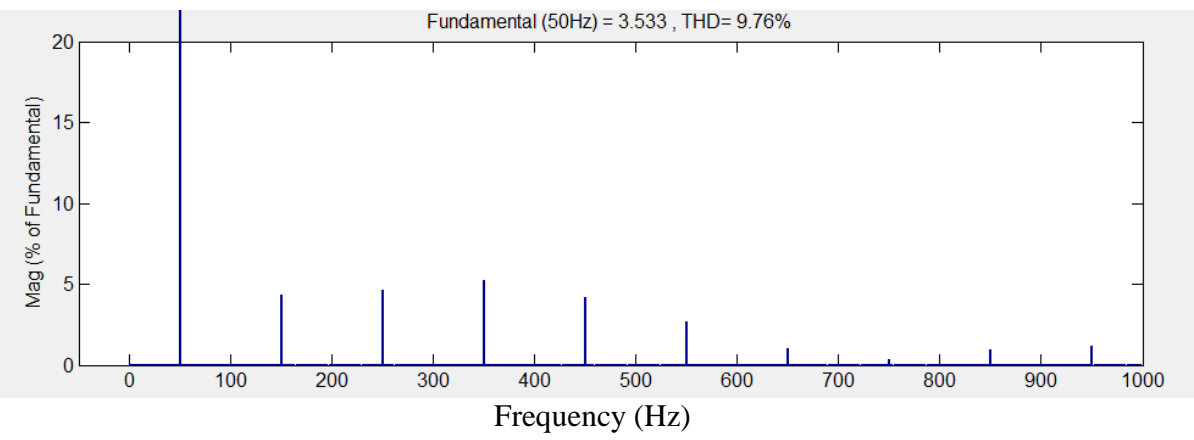

Figure 15. THD in the proposed control method

Figure14 and Figure15 show the THD diagram of $v_{i n}$ and $i_{i n}$ in PRN controller and PR controller.THD is reduced significantly and the power factor is improved greatly. The THD in PRN controller is $9.76 \%$, and the maximum power factor is $98.9 \%$. Furthermore, this paper lists THD in different control methods.

Table 1. THD in different power control methods.

\begin{tabular}{|c|c|}
\hline PI controller & $20.8 \%$ \\
\hline Repetitive method & $17.6 \%$ \\
\hline TL-QZSI method & $18.4 \%$ \\
\hline PR & $14.7 \%$ \\
\hline PRN & $9.76 \%$ \\
\hline
\end{tabular}

\section{Conclusions}

This paper analysed the electrolytic capacitors-less inverter system and proposed a novel power control method. The main conclusions can be summarized as follows: 1) the conventional method can't track the DC and AC component at the same time. In accurate power tracking causes the input 
current harmonics distortion. 2) In order to tracking power rapidly and accurately, this paper proposed a new inverter power controller (PRN) that can obviously reduce the THD of the source side and the amount of harmonic distortion, and improve the power factor. 3) The simulation results verified the validity of the proposed method. The input power factor is $98.9 \%$ and the THD is $9.76 \%$.

\section{Acknowledgements}

This work was supported by the Shenzhen Science and Technology Plan Project (Grant No : JCYJ20150403161923537

\section{References}

1. Ando, Itaru, et al. "Long life ups based on active filter and flywheel without electrolytic capacitor." Power Electronics and Applications, 2007 European Conference on. IEEE, (2007).

2. Yoo, Hyunjae, and Seung-Ki Sul. "A novel approach to reduce line harmonic current for a three-phase diode rectifier-fed electrolytic capacitor-less inverter." Applied Power Electronics Conference and Exposition, 2009. APEC 2009. Twenty-Fourth Annual IEEE. IEEE, (2009).

3. Haga, H., I. Takahashi, and K. Ohishi. "Direct torque IPM motor control method to obtain unity power factor using a single-phase diode rectifier." Electric Machines and Drives Conference, 2003. IEMDC'03. IEEE International. Vol. 2. IEEE, (2003).

4. Inazuma, Kazuya, Kiyoshi Ohishi, and Hitoshi Haga. "High-power-factor control for inverter output power of IPM motor driven by inverter system without electrolytic capacitor." Industrial Electronics (ISIE), 2011 IEEE International Symposium on. IEEE, (2011).

5. Utsugi, Hiroaki, Kiyoshi Ohishi, and Hitoshi Haga. "Reduction in current harmonics of electrolytic capacitor-less diode rectifier using inverter-controlled IPM motor." IECON 2012-38th Annual Conference on IEEE Industrial Electronics Society. IEEE, (2012).

6. Inazuma, Kazuya, et al. "High-power-factor single-phase diode rectifier driven by repetitively controlled IPM motor." Industrial Electronics, IEEE Transactions on 60.10 (2013): 4427-4437.

7. Zhou, Yufei, et al. "Electrolytic capacitor-less single-stage boost three-phase inverter for variable-speed AC motor system." Power Electronics and Drive Systems (PEDS), 2015 IEEE 11th International Conference on. IEEE, (2015).

8. Teodorescu, Remus, et al. "Proportional-resonant controllers and filters for grid-connected voltage-source converters." Electric Power Applications, IEE Proceedings. Vol. 153. No. 5. IET, (2006).

9. Vidal, Ana, et al. "Assessment and optimization of the transient response of proportional-resonant current controllers for distributed power generation systems." Industrial Electronics, IEEE Transactions on 60.4 (2013): 1367-1383. 